\title{
In vivo evaluation of a modified linear stapling device designed to facilitate accurate pathologic examination of the surgical margin
}

\author{
Hironori Tsujimoto ${ }^{1}$ Hitoshi Tsuda $^{2} \cdot$ Shuichi Hiraki $^{1} \cdot$ Shinsuke Nomura $^{1}$ • \\ Nozomi Ito ${ }^{1} \cdot$ Kyohei Kanematsu ${ }^{1} \cdot$ Hiroyuki Horiguchi ${ }^{1} \cdot$ Suefumi Aosasa $^{1}$ • \\ Junji Yamamoto ${ }^{1}$ Kazuo Hase ${ }^{1}$
}

Received: 8 April 2015/Accepted: 10 July 2015/Published online: 22 July 2015

(c) The International Gastric Cancer Association and The Japanese Gastric Cancer Association 2015

\begin{abstract}
Background Microscopic involvement of the resection margin could influence the long-term outcome of patients undergoing curative surgery for gastric cancer. Linear staplers, commonly used for gastrectomies, are often equipped with three lines of staples on either side of the resection line. Although multiple lines of staples reinforce closure of the gastric or intestinal stump, they could hinder accurate histopathologic evaluation of the surgical margin of the resected specimen.

Methods We modified a linear stapling device by removing one line (stapler E2) or two lines (stapler E1) of staples on the specimen side, and attempted to dissect a silicon film and then the stomach from a porcine model using the stapling device and examined the distances between the cutting edge and the nearest staple line.

Results The distance between the cutting edge and the staple line for stapler E1 was significantly greater than the distance between the cutting edge and the nearest staple line for stapler E2 or the control device. Consequently, specimens of exemplary quality were available for pathologic examination of the surgical margin. Moreover, the lack of multiple layers of staples did not result in contamination of the abdominal cavity with gastric juice during laparoscopic procedures in the porcine model.

Conclusions Stapler E1 is safe and could be useful for the pathologic evaluation of the true surgical margin.
\end{abstract}

Hironori Tsujimoto

tsujihi@ndmc.ac.jp

1 Department of Surgery, National Defense Medical College, 3-2 Namiki, Tokorozawa 359-8513, Japan

2 Department of Basic Pathology, National Defense Medical College, 3-2 Namiki, Tokorozawa 359-8513, Japan
Keywords Laparoscopic surgery · Pathologic examination $\cdot$ Clinical outcome $\cdot$ Surgical margin

\section{Introduction}

Recent guidelines from the Japanese Gastric Cancer Association recommend that a sufficient margin be ensured when one is determining the resection line in gastrectomy with curative intent, i.e., a proximal margin of at least $3 \mathrm{~cm}$ for T2 or deeper tumors with an expansive growth pattern (types 1 and 2) and $5 \mathrm{~cm}$ for those with an infiltrative growth pattern (types 3 and 4) [1]. For T1 tumors, a gross resection margin of $2 \mathrm{~cm}$ should be obtained. When these guidelines cannot be observed, examination of the resection margin by intraoperative frozen section analysis is recommended.

Linear stapling devices are frequently used for dividing tissues and creating an anastomosis in laparoscopic surgical procedures [2,3]. To examine the surgical margin obtained with linear staplers, all staples must be removed or use of stapled tissues should be abandoned. However, it is very difficult to remove all staples from the tissue, and it is difficult to perform an accurate pathologic examination because of tissue damage. In addition, if the stapled tissue is not used, the true marginal tissue cannot be evaluated, which may lead to a false positive result.

Here, we report a novel modification of a linear stapling device, which removes the staples beforehand, and which was used for the pathologic evaluation of the true surgical margin, and we examine the feasibility and accuracy of the instrument for pathologic examination of the true margin. 


\section{Materials and methods}

\section{Setup for the linear stapling device}

An ECHELON FLEX ${ }^{\mathrm{TM}}$ Powered ENDOPATH ${ }^{\circledR}$ stapler (60 mm; Ethicon, Tokyo, Japan) was used. The cartridge cover was removed, and the staples from one line (stapler E2) or two lines (stapler E1) were removed with sharp Péan forceps (Fig. 1). We then replaced the cover. This maneuver can be completed in 1-2 min. The stapler cartridge was attached to the linear stapling device, which was then fired such that the side with the staples removed was used for pathologic examination. An original stapling device with all lines of staples intact served as a control.

\section{Measurement of the distance between the cutting edge and staple lines}

We used the stapler to resect a porcine stomach and a 3 mm-thick silicon film (Tanac, Gifu, Japan), and measured the distance between the cutting edge and staple lines using staplers E1 and E2 and the control device. Each experiment was performed independently three times.

\section{Feasibility of the stapling device in a porcine model}

A female pig was used to assess stapler E1. The pig was quarantined and acclimated in the animal facility for 1 week before surgery. General anesthesia was then maintained with $2 \%$ sevoflurane with the pig in the supine position. The intra-abdominal cavity was insufflated at a pressure of $12 \mathrm{mmHg}$ with the open technique. Under laparoscopic imaging, four additional $12-\mathrm{mm}$ ports were inserted into the upper left, lower right, lower left, and upper right quadrants. Laparoscopic partial gastrectomy

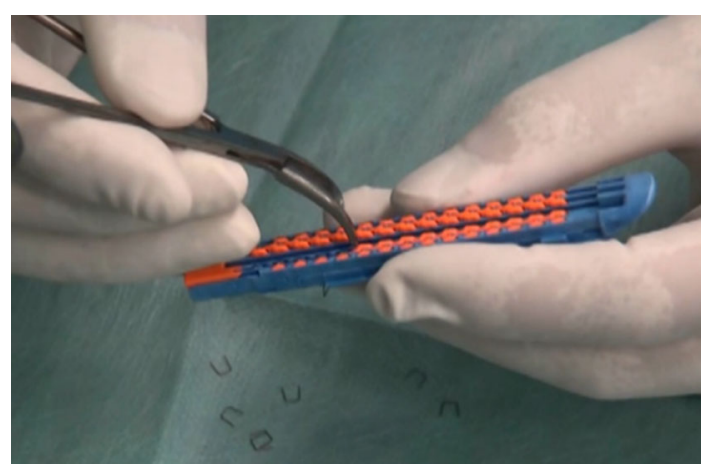

Fig. 1 Setup for the linear stapling device. An ECHELON FLEX ${ }^{\mathrm{TM}}$ Powered ENDOPATH ${ }^{\circledR}$ stapler (60 mm; Ethicon, Tokyo, Japan) was used. The cartridge cover was removed, and one line (stapler E2) or two lines (stapler E1) of staples were removed with sharp Péan forceps. Then, the cover was replaced, and the cartridge was attached to the stapling device was performed with stapler E1 for lesions assumed to be located in the anterior wall [4]. After partial gastrectomy, the blood vessels around the duodenum were occluded with an ultrasonically activated sealing device (Harmonic $\mathrm{ACE}^{\circledR}$ scalpel; Ethicon, Tokyo, Japan). After complete lateral mobilization of the duodenum, stapler E1 was applied to dissect the duodenum.

The resected specimens were fixed with $10 \%$ formaldehyde solution for $48 \mathrm{~h}$, embedded in paraffin, and cut into $5-\mu \mathrm{m}$ sections, which were stained with hematoxylin-eosin. The study protocol was approved by the Ethics Committee of the National Defense Medical College.

\section{Statistical analysis}

The results are presented as mean \pm standard deviation of the mean. Analysis of variance was used to analyze the distance between the cutting edge and the staple lines. All analyses were performed with StatView version 5.0 (SAS Institute, Cary, NC, USA). $P$ values less than 0.05 were considered statistically significant.

\section{Results}

Distance between the cutting edge and the nearest staple line for staplers E1 and E2 and the control device

The silicon film (data not shown) and the resected porcine stomach (Fig. 2) were successfully divided and stapled with all three staplers. There were significant differences in the distances between the cutting edge and the nearest staple line among the three staplers (Fig. 3). The distance between the cutting edge and the staple line for the resected porcine stomach and silicon film obtained with stapler E1 was $2.96 \pm 0.09 \mathrm{~mm}$ and $3.20 \pm 0.12 \mathrm{~mm}$, respectively, which should be of sufficient length to perform pathologic examinations.

\section{In vivo use of the proposed stapling device}

No gastric juice was spilled during surgery with the porcine model. Macroscopic photographs of the margins of the stomach and duodenum, which were divided with stapler E1, are shown in Fig. 4. The margin lengths were sufficient for macroscopic pathologic examination. Microscopic findings of the porcine duodenum dissected with stapler E1 are depicted in Fig. 5. All layers are depicted, which allowed a detailed pathologic examination of the true margins. 


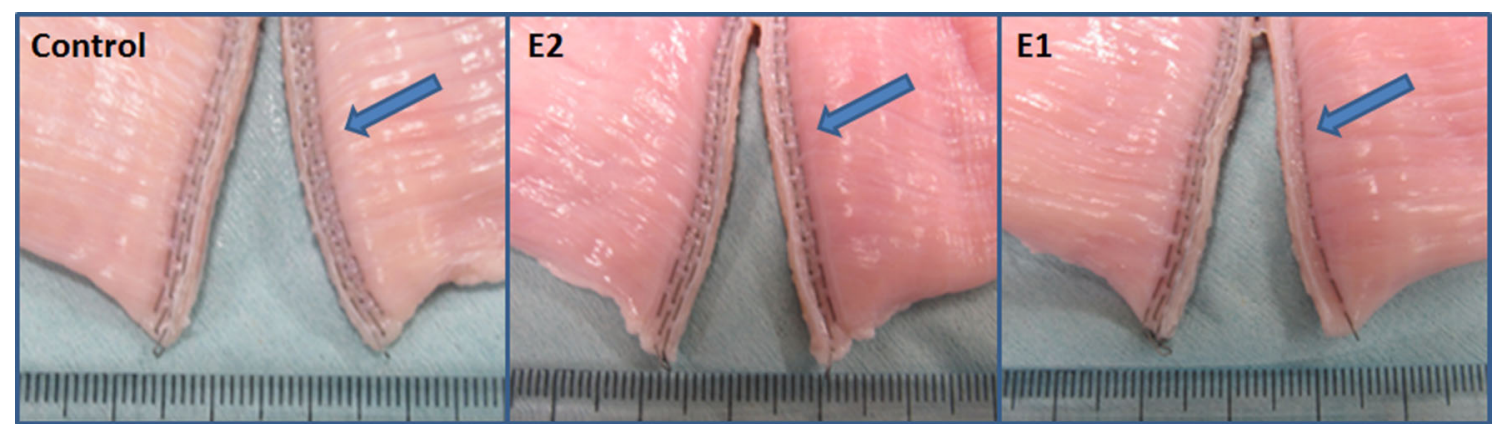

Fig. 2 Photographs of a porcine stomach resected with each of the three linear stapling devices (stapler E1, stapler E2, and control). The resected porcine stomach was successfully divided and stapled with all three staplers

Fig. 3 The distance between the cutting edge and the nearest staple line in the resected porcine stomach and silicon film. There were significant differences in the distance between the cutting edge and the nearest staple line among the three staplers in both the resected porcine stomach and the silicon film. Asterisk $P<0.05$ between the two groups
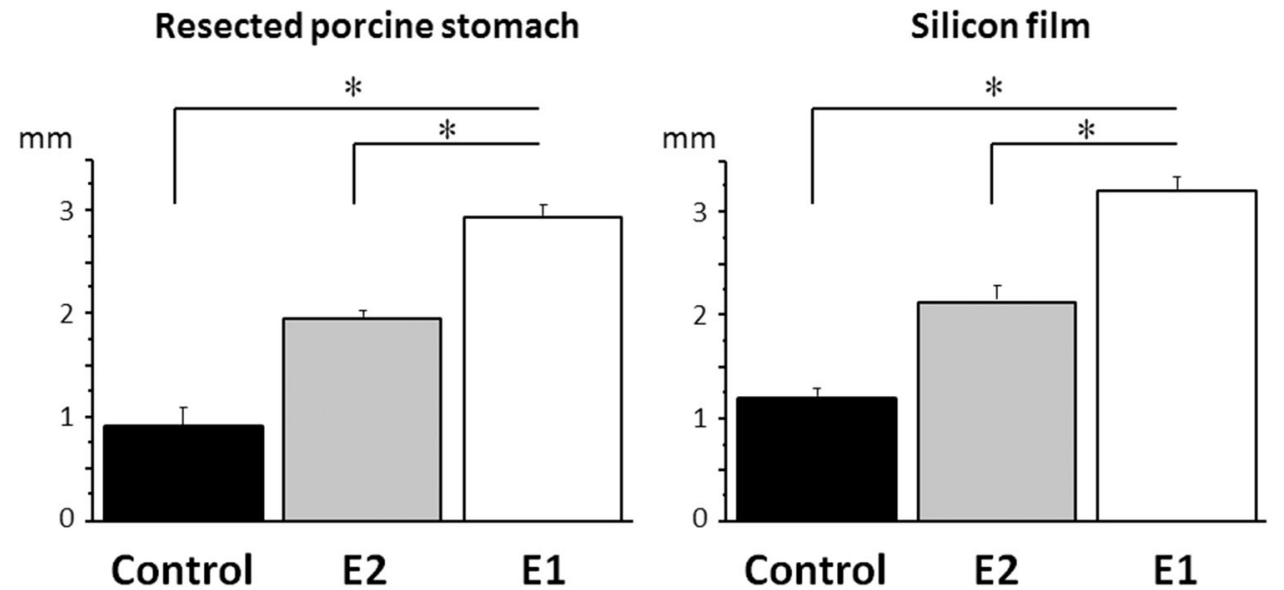

Fig. 4 Photographs showing a sufficient margin length for pathologic examination after partial gastrectomy (a) and duodenal dissection (b) in the porcine model performed with stapler E1. Magnification $\times 40$
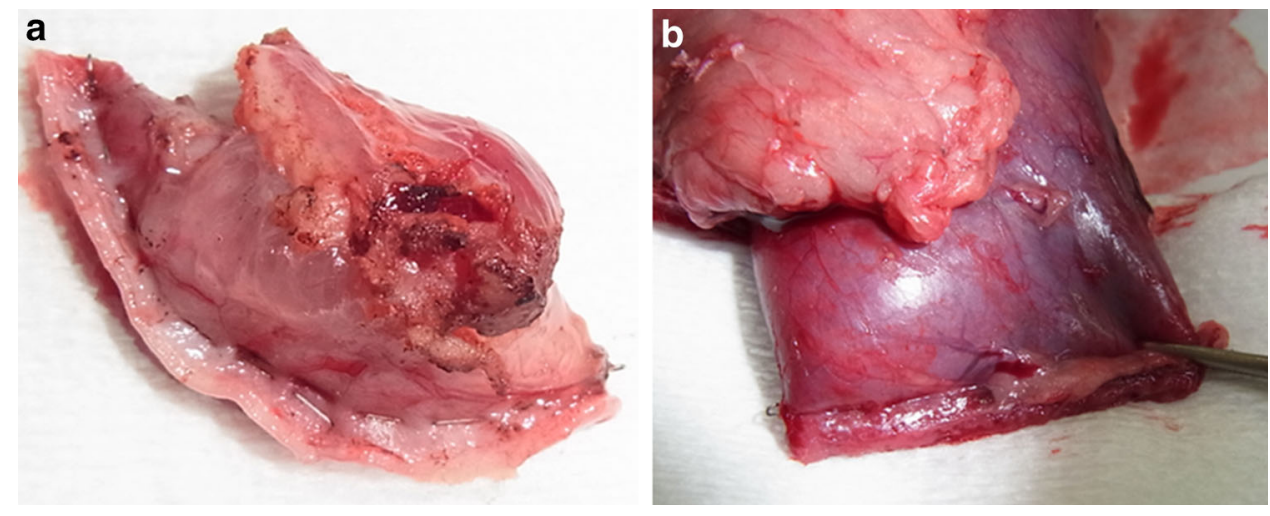

\section{Discussion}

We modified a linear stapling device to accurately evaluate the surgical margins and assessed the efficacy and clinical advantage of the device.

Many surgeons have noted that the microscopic involvement of a positive margin influences long-term outcomes, particularly in the early stage of gastric cancer, even if radical gastrectomy was completed. However, many reports regarding the clinical importance of a positive margin did not describe the method of evaluation of the true surgical margin or the most appropriate device to dissect the tissue. Although a positive margin has often been associated with poor prognosis in gastric cancer [5-8], it has lost prognostic relevance in some specific cohorts [9-11]. Besides the biological characteristics of these specific cohorts, inaccurate handling of the surgical margin for histologic evaluation may have led to these conflicting results.

Linear stapling instruments are frequently used to divide the duodenum and stomach during gastrectomy. These 


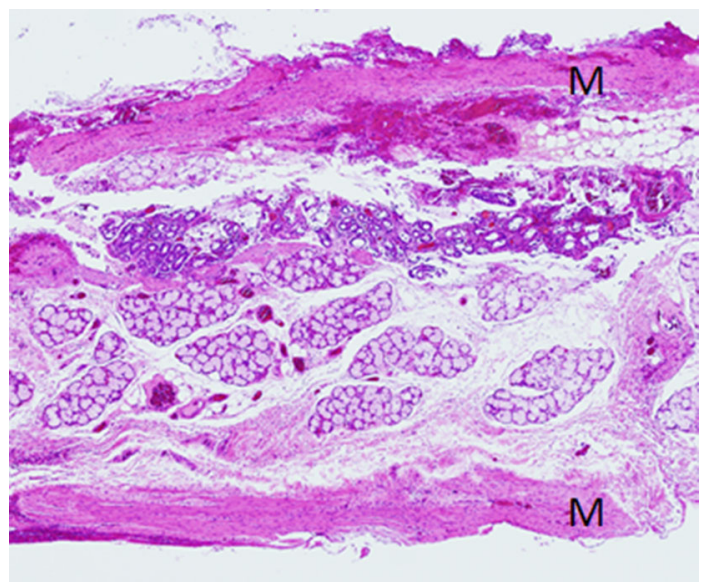

Fig. 5 Microscopic examination of the duodenal margin in the pig. All layers of the duodenum were visible on microscopic examination, suggesting the feasibility of the pathologic examination of all layers. $M$ muscular layer

devices generally have four or six rows of staples and form two or three staple lines on a margin with a length of approximately $4-5 \mathrm{~mm}$. Because these staplers were originally developed to set irremovable staples, it can be very difficult to remove all staples without damaging the tissue to facilitate pathologic evaluation of the margin. In addition, if pathologic evaluation of this site is not possible, this may lead to a false positive result, and thus reoperation should be considered. Accordingly, accurate pathologic evaluation of the true margin without tissue damage is crucial to assess the clinical significance of a positive margin and for additional intervention [12].

We demonstrated that stapler E1 was useful for laparoscopic partial gastrectomy and dividing the duodenum in the porcine model, which included all layers. Although only one line of staples was applied to the proximal stump, the staple placement was sufficient to prevent intraoperative leakage of gastric juice.

Because of the difference in the intensity between the mucosa and muscularis propria, the tissue containing the margin may retract, rendering it difficult to evaluate all layers in the clinical setting. Hence, use of a bioabsorbable sleeve (Gunze, Ayabe, Japan) to reinforce the staples may be helpful to prevent the tissue stump from "rolling up" [13].

In conclusion, the proposed linear stapling device in which staples are removed beforehand is safe and could be useful for the pathologic evaluation of the true surgical margin.

Acknowledgments The authors thank Enago (http://www.enago.jp/) for the English language review.

\section{Compliance with ethical standards}

Ethical standards The study protocol was reviewed and approved by the Institutional Review Board of the National Defense Medical College (Tokorozawa, Japan).

Conflict of interest The authors declare that they have no commercial associations that might pose a conflict of interest in connection with the submitted article.

\section{References}

1. Japanese Gastric Cancer Association. Japanese gastric cancer treatment guidelines 2010 (ver. 3). Gastric Cancer. 2011;14:113-23.

2. Tsujimoto H, Uyama I, Yaguchi Y, Kumano I, Takahata R, Matsumoto Y, et al. Outcome of overlap anastomosis using a linear stapler after laparoscopic total and proximal gastrectomy. Langenbecks Arch Surg. 2012;397:833-40.

3. Uyama I, Sugioka A, Matsui H, Fujita J, Komori Y, Hatakawa Y, et al. Laparoscopic side-to-side esophagogastrostomy using a linear stapler after proximal gastrectomy. Gastric Cancer. 2001;4:98-102.

4. Inoue H, Ikeda H, Hosoya T, Yoshida A, Onimaru M, Suzuki M, et al. Endoscopic mucosal resection, endoscopic submucosal dissection, and beyond: full-layer resection for gastric cancer with nonexposure technique (CLEAN-NET). Surg Oncol Clin N Am. 2012;21:129-40.

5. Kim SH, Karpeh MS, Klimstra DS, Leung D, Brennan MF. Effect of microscopic resection line disease on gastric cancer survival. J Gastrointest Surg. 1999;3:24-33.

6. Nagata T, Ichikawa D, Komatsu S, Inoue K, Shiozaki A, Fujiwara $\mathrm{H}$, et al. Prognostic impact of microscopic positive margin in gastric cancer patients. J Surg Oncol. 2011;104:592-7.

7. Woo JW, Ryu KW, Park JY, Eom BW, Kim MJ, Yoon HM, et al. Prognostic impact of microscopic tumor involved resection margin in advanced gastric cancer patients after gastric resection. World J Surg. 2014;38:439-46.

8. Morgagni P, Garcea D, Marrelli D, de Manzoni G, Natalini G, Kurihara $\mathrm{H}$, et al. Does resection line involvement affect prognosis in early gastric cancer patients? An Italian multicentric study. World J Surg. 2006;30:585-9.

9. Kodera Y, Yamamura Y, Ito S, Kanemitsu Y, Shimizu Y, Hirai T, et al. Is Borrmann type IV gastric carcinoma a surgical disease? An old problem revisited with reference to the result of peritoneal washing cytology. J Surg Oncol. 2001;78:175-81.

10. Piessen G, Messager M, Leteurtre E, Jean-Pierre T, Mariette C. Signet ring cell histology is an independent predictor of poor prognosis in gastric adenocarcinoma regardless of tumoral clinical presentation. Ann Surg. 2009;250:878-87.

11. Shiraishi N, Adachi Y, Kitano S, Bandoh T, Inomata M, Yasuda $\mathrm{K}$, et al. Gastric cancer with extragastric lymph node metastasis: multivariate prognostic study. Gastric Cancer. 2000;3:211-8.

12. Raziee HR, Cardoso R, Seevaratnam R, Mahar A, Helyer L, Law $\mathrm{C}$, et al. Systematic review of the predictors of positive margins in gastric cancer surgery and the effect on survival. Gastric Cancer. 2012;15:S116-24.

13. Saito Y, Omiya H, Shomura Y, Minami K, Imamura H. A new bioabsorbable sleeve for staple-line reinforcement: report of a clinical experience. Surg Today. 2002;32:297-9. 